\title{
Significance of Ureteroscopic Biopsy Grade in Patients with Upper Tract Urothelial Carcinoma
}

\author{
Junya Furukawa Hideaki Miyake Iori Sakai Masato Fujisawa \\ Division of Urology, Kobe University Graduate School of Medicine, Kobe, Japan
}

\section{Key Words}

Upper tract urothelial carcinoma • Ureteroscopic biopsy • Urinary cytology

\begin{abstract}
Background: The objective of this study was to assess the significance of the ureteroscopic biopsy grade for patients with upper tract urothelial carcinoma (UTUC). Patients and Methods: This study included 40 patients who were diagnosed with a single focus of UTUC by ureteroscopic biopsy and subsequently underwent nephroureterectomy. The significance of the biopsy grade as a predictive factor for pathological outcomes of nephroureterectomy was retrospectively analyzed. Results: Of these 40 patients, 19 (47.5\%) and 21 (52.5\%) were diagnosed with low and high grade UTUC, respectively. The ureteroscopic biopsy grade matched the pathological grade of surgically resected specimens in 35 of the 40 cases $(87.5 \%)$, and there was a significant correlation between the biopsy and pathological grades ( $p<0.001$ ). Furthermore, the biopsy grade was also shown to be closely associated with the pathological stage ( $p<0.001)$; that is, only 1 of the 19 patients (5.3\%) with biopsy low grade UTUC were pathologically diagnosed as having muscle invasive disease, while 17 of the 21 patients (81.0\%) with biopsy high grade UTUC appeared to show tumor invasion into muscle or deeper. Conclusions: The grade of UTUC on ureteroscopic biopsy could provide accurate diagnostic information on the final pathology of nephroureterectomy specimens.
\end{abstract}

Copyright $\odot 2012$ S. Karger AG, Basel

\section{KARGER}

Fax +4161306 1234

E-Mail karger@karger.ch

www.karger.com
(C) 2012 S. Karger AG, Basel

$1015-9770 / 12 / 0063-0156 \$ 26.00 / 0$

Accessible online at:

www.karger.com/cur

\section{Introduction}

Upper tract urothelial carcinoma (UTUC) is a relatively rare disease accounting for approximately $5 \%$ of malignant urothelial tumors [1]. Although nephroureterectomy with excision of a cuff of the bladder mucosa is currently regarded as the standard for the management of nonmetastatic UTUCs, prognostic outcomes following nephroureterectomy have been shown to vary depending on major pathological factors, particularly the tumor grade and pathological stage [2-5]. For example, Hall et al. [2] reported that when evaluated according to the pathological stage, 5-year survival rates in patients with UTUC undergoing nephroureterectomy ranged from $100 \%$ for pTa disease to $41 \%$ for pT3 disease, while our previous study demonstrated a significant difference in postoperative cancer-specific survival between patients with low grade UTUC and those with G3 UTUC [3]. However, during the last two decades, more conservative approaches to the treatment of UTUC, such as segmental ureteral resection and transureteral endoscopic management, have gained acceptance for patients requiring a nephron-sparing approach, including those with a solitary kidney, bilateral UTUC, renal impairment or significant comorbidities [6, 7]. Furthermore, some recent studies recommended such conservative managements for selected patients with small, low grade UTUC and a normal contralateral kidney $[8,9]$.

Considering the wide variety of surgical approaches for UTUC and their complicated indications, it is crucial 
to obtain precise information regarding the characteristics of the primary tumor prior to surgical resection in order to determine the appropriate therapeutic option for each patient with UTUC. In this study, therefore, we retrospectively reviewed our experience with ureteroscopic biopsy for UTUC, and evaluated its significance focusing on the biopsy grade as a predictive factor for pathological outcomes of nephroureterectomy specimens.

\section{Patients and Methods}

This study included a total of 40 consecutive patients who were diagnosed with a single focus of UTUC based on the findings on diagnostic ureteroscopy and biopsy, and were subsequently treated with nephroureterectomy at our institution between 2001 and 2009. During this interval, there were additional 87 patients with a single focus of UTUC who underwent nephroureterectomy without an examination by diagnostic ureteroscopy. In this study, patients with multiple tumor foci in the urinary tract were excluded for performing a rigid comparison of primary tumor characteristics between biopsy and surgically resected specimens.

The upper urinary tract was examined using rigid $11.5 \mathrm{Fr}$ and/ or flexible $9 \mathrm{Fr}$ ureteroscopes, and biopsies of suspicious lesions were performed with 3 Fr cold cup forceps. All patients underwent nephroureterectomy within 3 months of ureteroscopic biopsy using either a standard open or laparoscopic approach, including the removal of the kidney, entire length of the ureter and adjacent segment of the bladder cuff, as previously described [3]. In this series, it was determined by preoperative clinical status whether regional lymphadenectomy was performed; however, the range of dissection was not uniformly determined. All specimens obtained from biopsy and nephroureterectomy were graded based on the 2004 World Health Organization classification and staged according to the 2002 TNM classification system by a pathologist.

Statistical analyses by Pearson's correlation coefficient for comparing pathological findings between biopsy and surgically resected specimens were carried out using Statview 5.0 software (Abacus Concepts, Inc., Berkeley, CA, USA). Probability (p) values less than 0.05 were considered significant.

\section{Results}

Clinicopathological characteristics of the 40 patients included in this study are summarized in table 1 . All 40 patients were examined by upper tract urinary cytology, and positive findings were obtained in 15 (37.5\%). This outcome was significantly associated with the ureteroscopic biopsy grade $(\mathrm{p}=0.041)$; that is, a positive upper tract cytology was demonstrated in 4 of 19 patients with biopsy low grade disease $(21.1 \%)$ and 11 of 21 with biopsy high grade disease (52.4\%).

As shown in table 2, there was a significant association between the tumor grade of ureteroscopic biopsy and that

Ureteroscopic Biopsy for Upper Tract

Urothelial Carcinoma
Table 1. Patient characteristics

\begin{tabular}{lr}
\hline Item & Value \\
\hline $\begin{array}{l}\text { Median age (range), years } \\
\text { Gender, } \%\end{array}$ & $70.0(52-89)$ \\
$\quad$ Male & $35(87.5)$ \\
$\quad$ Female & $5(12.5)$ \\
Tumor location, $\%$ & \\
$\quad$ Renal pelvis & $19(47.5)$ \\
$\quad$ Ureter & $21(52.5)$ \\
Tumor site, $\%$ & \\
$\quad$ Right & $11(27.5)$ \\
Left & $29(72.5)$ \\
Urinary cytology, $\%$ & \\
$\quad$ Negative & $15(37.5)$ \\
Positive & $25(62.5)$ \\
Ureteroscopic biopsy grade, $\%$ & \\
$\quad$ Low & $19(47.5)$ \\
High & $21(52.5)$ \\
Pathological grade, $\%$ & \\
$\quad$ Low & $14(35.0)$ \\
High & $26(65.0)$ \\
Pathological stage, $\%$ & \\
pTa & $13(32.5)$ \\
pT1 & $9(22.5)$ \\
pT2 & $6(15.0)$ \\
pT3 & $12(30.0)$ \\
\hline
\end{tabular}

Table 2. Association between biopsy and pathological grade and stage

\begin{tabular}{lccc}
\hline & \multicolumn{2}{c}{ Biopsy grade } & $\mathrm{p}$ \\
\cline { 2 - 3 } & Low & High & \\
\hline $\begin{array}{l}\text { Pathological grade, } \% \\
\text { Low }\end{array}$ & $14(100)$ & $0(0)$ & $<0.001$ \\
$\quad$ High & $5(19.2)$ & $21(80.8)$ & \\
$\begin{array}{l}\text { Pathological stage, } \% \\
\text { pTa }\end{array}$ & $13(100)$ & $0(0)$ & $<0.001$ \\
pT1 & $5(55.6)$ & $4(44.4)$ & \\
pT2 & $1(16.7)$ & $5(83.3)$ & \\
pT3 & $0(0)$ & $12(100)$ & \\
\hline
\end{tabular}

of the nephroureterectomy specimen $(\mathrm{p}<0.001)$. Ureteroscopic biopsy grades were identical to the pathological grade of surgically resected specimens in 35 of the 40 cases $(87.5 \%)$; however, despite no upgraded cases, ureteroscopic biopsy downgraded 5 of 26 patients with high grade disease (19.2\%). 
The relation between the ureteroscopic biopsy grade and pathological stage of the nephroureterectomy specimen was then assessed (table 2). In these 40 patients, there was a significant association between these two factors $(p<0.001)$. In particular, there was only 1 patient with pathologically diagnosed muscle invasive disease in 19 patients with biopsy low grade UTUC (5.3\%), and 17 of 21 patients with biopsy high grade UTUC $(81.0 \%)$ were diagnosed with a tumor invading the muscle or deeper.

In this series, 20 patients $(50.0 \%)$ received regional lymphadenectomy in addition to nephroureterectomy. There was no patient with biopsy low grade UTUC who were diagnosed with pathologically positive lymph node, while 4 of the 13 patients with biopsy high grade UTUC $(30.8 \%)$ appeared to be positive for nodal involvement. Of these 20, there was no significant association between the ureteroscopic biopsy grade and nodal stage.

\section{Discussion}

Although the therapeutic strategy for patients with UTUC remains under development, various options for the treatment of this disease have become available [1, 6-9]. However, in order to provide suitable treatment for each patient with UTUC, it is necessary to obtain accurate information on the clinicopathological features of the primary tumors preoperatively. In fact, a conservative approach has been shown to be a reasonable alternative to nephroureterectomy for properly selected patients with low-risk UTUC [6-9]. In addition, if muscle invasive and/or non-organ-confined disease is preoperatively diagnosed, several additional options, such as neoadjuvant chemotherapy and extensive lymphadenectomy, could be performed [10]. Considering these findings, we retrospectively analyzed the significance of ureteroscopic biopsy as an indicator for pathological outcomes of nephroureterectomy specimens from patients with UTUC.

This study included a total of 40 patients who were diagnosed with UTUC by ureteroscopic biopsy, and subsequently underwent nephroureterectomy. The reliability of ureteroscopic biopsy for the staging of UTUC is controversial, since diagnostic accuracy requires access to the base of the tumor, which is not always possible [11]; accordingly, we focused on the biopsy tumor grade, and evaluated its association with the final pathological findings. There was a significant relation between the tumor grade of ureteroscopic biopsy and that of the nephroureterectomy specimen; however, high grade dis- ease represented a comparatively heterogeneous group, approximately $20 \%$ of which could not be exactly graded by ureteroscopic biopsy. Furthermore, the pathological stage also showed a significant association with the ureteroscopic biopsy grade. In particular, the presence of muscle invasion could be satisfactory differentiated by ureteroscopic biopsy irrespective of biopsy tumor grade. Collectively, these findings suggest that the grading of UTUC by ureteroscopic biopsy could provide useful diagnostic information on the final pathology of resected specimens.

It would be of interest to develop a system that more accurately predicts the final pathological findings in nephroureterectomy specimens prior to surgery. Considering the insufficient ability to predict the final pathology using a single modality, the utility of multivariable combination model of plural modalities has been investigated $[1,10-12]$. For example, Favaretto et al. [10] reported a preoperative model that facilitates accurate staging of UTUC based on the findings of readily available imaging examinations and ureteroscopic parameters, while Skolarikos et al. [11] showed that, for patients with G2 biopsies, the combined use of the urinary cytology and biopsy grade resulted in the improvement of the sensitivity and specificity of high-grade UTUC detection. Furthermore, several recent studies suggested the usefulness of molecular markers that were shown to be associated with pathological factors in nephroureterectomy specimens $[5,13,14]$; therefore, it may help to obtain diverse information by examining the expression of such molecules in ureteroscopic biopsy specimens.

In conclusion, we recommend performing ureteroscopic biopsy for patients with suspicious findings of UTUC in order to determine an optimal therapeutic strategy for each patient prior to surgical treatment, since the outcomes of ureteroscopic biopsy, particularly biopsy grade, could provide useful information for the prediction of the final pathological findings of nephroureterectomy specimens. However, the ureteroscopy biopsy grade alone has limited ability to individualize treatment options for patients with UTUC; therefore, the incorporation of multiple validated modalities, such as cytology and radiological examinations, into the findings of ureteroscopic biopsy might help guide decision-making for selecting treatment for patients with UTUC. 


\section{References}

1 Painter DJ, Timoney AG, Denton K, Alken P, Keeley FX Jr: The modern management of upper urinary tract urothelial cancer: tumour diagnosis, grading and staging. BJU Int 2007; 99:973-977.

$>2$ Hall MC, Womack S, Sagalowsky AI, Carmody T, Erickstad MD, Roehrborn CG: Prognostic factors, recurrence, and survival in transitional cell carcinoma of the upper urinary tract: a 30 -year experience in 252 patients. Urology 1998;52:594-601.

-3 Terakawa T, Miyake H, Hara I, Takenaka A, Fujisawa M: Retroperitoneoscopic nephroureterectomy for upper urinary tract cancer: a comparative study with conventional open retroperitoneal nephroureterectomy. J Endourol 2008;22:1693-1699.

-4 Margulis V, Youssef RF, Karakiewicz PI, Lotan Y, Wood CG, Zigeuner R, Kikuchi E, Weizer A, Raman JD, Remzi M, Roscigno M, Montorsi F, Bolenz C, Kassouf W, Shariat SF: Preoperative multivariable prognostic model for prediction of nonorgan confined urothelial carcinoma of the upper urinary tract. J Urol 2010;184:453-458.

5 Chromecki TF, Bensalah K, Remzi M, Verhoest G, Cha EK, Scherr DS, Novara G, Karakiewicz PI, Shariat SF: Prognostic factors for upper urinary tract urothelial carcinoma. Nat Rev Urol 2011;8:440-447.
6 Martinez-Pineiro JA, Garcia Matres MJ, Martinez-Pineiro L: Endourological treatment of upper tract urothelial carcinomas: analysis of a series of 59 tumors. J Urol 1996;156: 377-385.

7 Grasso M, Fraiman M, Levine M: Ureteropyeloscopic diagnosis and treatment of upper urinary tract urothelial malignancies. Urology 1999;54:240-246.

8 Thompson RH, Krambeck AE, Lohse CM, Elliott DS, Patterson DE, Blute ML: Endoscopic management of upper tract transitional cell carcinoma in patients with normal contralateral kidneys. Urology 2008;71:713-717.

-9 Sowter SJ, Ilie CP, Efthimiou I, Tolley DA: Endourologic management of patients with upper-tract transitional-cell carcinoma: longterm follow-up in a single center. J Endourol 2007;21:1005-1009.

10 Favaretto RL, Shariat SF, Savage C, Godoy G, Chade DC, Kaag M, Bochner BH, Coleman J, Dalbagni G: Combining imaging and ureteroscopy variables in a preoperative multivariable model for prediction of muscle-invasive and non-organ confined disease in patients with upper tract urothelial carcinoma. BJU Int 2012;109:77-82.
11 Skolarikos A, Griffiths TR, Powell PH, Thomas DJ, Neal DE, Kelly JD: Cytologic analysis of ureteral washings is informative in patients with grade 2 upper tract TCC considering endoscopic treatment. Urology 2003;61:1146-1150.

12 Margulis V, Shariat SF, Matin SF, Kamat AM, Zigeuner R, Kikuchi E, Lotan Y, Weizer A, Raman JD, Wood CG: Outcomes of radical nephroureterectomy: a series from the Upper Tract Urothelial Carcinoma Collaboration. Cancer 2009;115:1224-1233.

13 Winkler A, Zigeuner R, Rehak P, Hutterer G, Chromecki T, Langner C: CD24 expression in urothelial carcinoma of the upper urinary tract correlates with tumour progression. Virchows Arch 2007;450:59-64.

14 Muramaki M, Miyake H, Terakawa T, Kusuda Y, Fujisawa M: Expression profile of Ecadherin and $\mathrm{N}$-cadherin in urothelial carcinoma of the upper urinary tract is associated with disease recurrence in patients undergoing nephroureterectomy. Urology 2011;78: 1443.e7-12. 